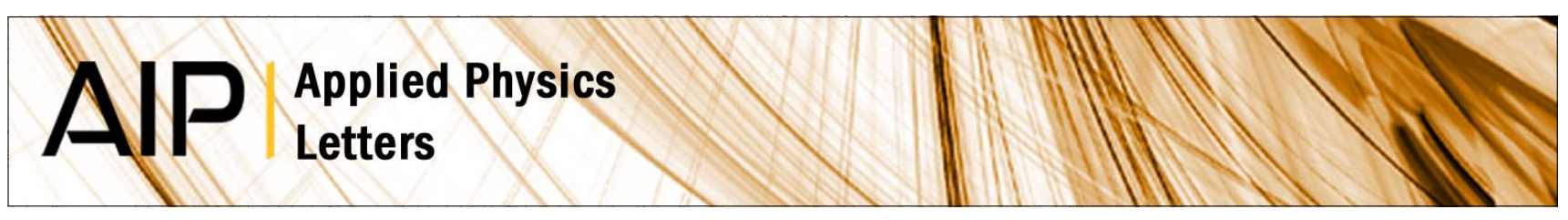

\title{
Turn-key module for neutron scattering with sub-micro-eV resolution
}

R. Georgii, G. Brandl, N. Arend, W. Häußler, A. Tischendorf et al.

Citation: Appl. Phys. Lett. 98, 073505 (2011); doi: 10.1063/1.3556558

View online: http://dx.doi.org/10.1063/1.3556558

View Table of Contents: http://apl.aip.org/resource/1/APPLAB/v98/i7

Published by the American Institute of Physics.

Additional information on Appl. Phys. Lett.

Journal Homepage: http://apl.aip.org/

Journal Information: http://apl.aip.org/about/about_the_journal

Top downloads: http://apl.aip.org/features/most_downloaded

Information for Authors: http://apl.aip.org/authors

\section{ADVERTISEMENT}

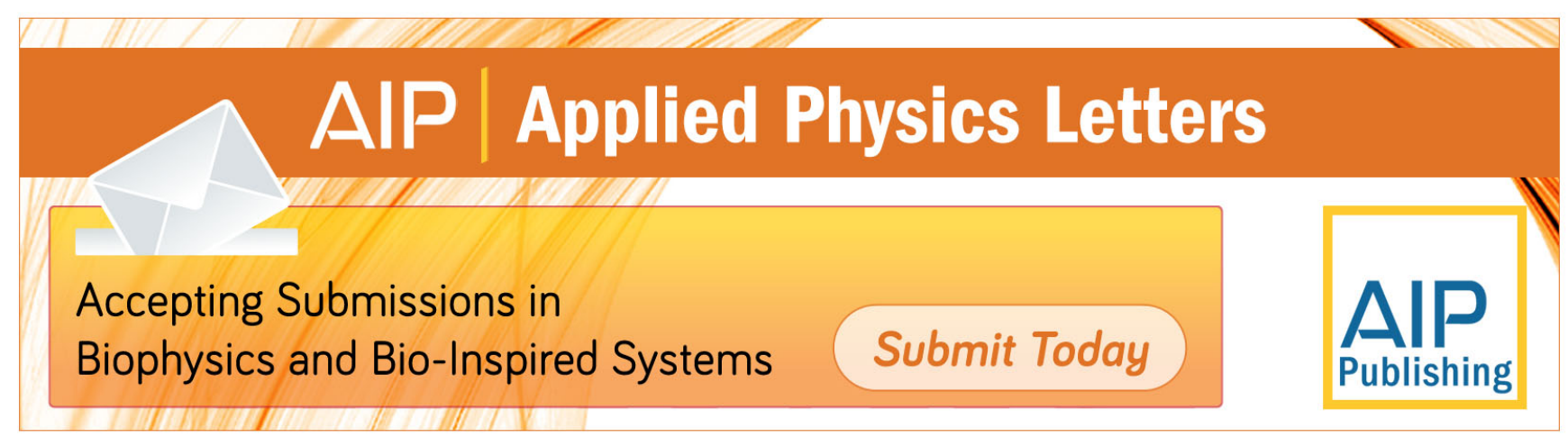




\title{
Turn-key module for neutron scattering with sub-micro-eV resolution
}

\author{
R. Georgii, ${ }^{1,2, a)}$ G. Brandl, ${ }^{1,2}$ N. Arend, ${ }^{2,3}$ W. Häußler, ${ }^{1,2}$ A. Tischendorf, ${ }^{2}$ C. Pfleiderer, ${ }^{2}$ \\ P. Böni, ${ }^{2}$ and J. Lal ${ }^{4}$ \\ ${ }^{1}$ Forschungsneutronenquelle Heinz-Maier Leibnitz, Technische Universität München, \\ 85748 Garching, Germany \\ ${ }^{2}$ Physik-Department E21, Technische Universität München, 85748 Garching, Germany \\ ${ }^{3}$ Forschungszentrum Jülich GmbH, JCNS-1 \& ICS-1, Oak Ridge National Laboratory, Oak Ridge, \\ Tennessee 37831, USA \\ ${ }^{4}$ Materials Science Division, Argonne National Lab, Argonne, Illinois 60439, USA
}

(Received 3 December 2010; accepted 27 January 2011; published online 18 February 2011)

\begin{abstract}
We report the development of a compact turn-key module that boosts the resolution in quasielastic neutron scattering by several orders of magnitude down to the low sub-micro-eV range. It is based on a pair of neutron resonance spin flippers that generate a well defined temporal intensity modulation, also known as Modulation of IntEnsity by Zero Effort (MIEZE). The module may be used under versatile conditions, in particular, in applied magnetic fields and for depolarizing and incoherently scattering samples. We demonstrate the power of MIEZE in studies of the helimagnetic order in MnSi under applied magnetic fields. (c) 2011 American Institute of Physics.
\end{abstract}

[doi:10.1063/1.3556558]

Neutron scattering is an extremely powerful technique for studies of the dynamical properties of condensed matter systems. Prominent examples of great current interest concern the spin dynamics in transition metal and rare earth compounds and diffusive processes in soft matter systems such as proteins, liquid crystals, and emulsions. A precondition to unravel some of the most important scientific challenges is the need for high energy and momentum resolution.

Conventional neutron scattering techniques such as triple-axis and time-of-flight spectroscopy provide momentum resolved energy resolutions of the order of $\simeq 10 \mu \mathrm{eV}$. Backscattering reaches the sub-micro-eV regime, however, sacrificing momentum resolution. This is contrasted by neutron spin-echo (NSE) methods, ${ }^{1,2}$ which offer high energy and momentum resolutions in the low sub-micro-eV rangeseveral orders of magnitude below the typical resolutions of conventional techniques. However, because NSE scattering uses polarized neutrons it is inherently sensitive to the depolarization of the neutron beam. Therefore, it is technically very demanding to perform NSE measurements under applied magnetic fields or in depolarizing samples ${ }^{3,4}$ such as superconductors, ferromagnets, ${ }^{5}$ or protonated soft matter systems.

In this letter, we report the development of a turn-key module, the MIEZE (Modulation of IntEnsity by Zero Effort) box shown in Fig. 1. In combination with a polarizer, a polarizing analyzer, and a fast detector this box allows to improve the energy resolution in all types of neutron scattering instruments capable of studying quasielastic scattering, notably diffractometers, small-angle neutron scattering cameras, and reflectometers (especially instruments for small $q$ and cold neutrons), down to the sub-micro-eV range. The module is based on the so-called MIEZE-I technique (Modulation of IntEnsity by Zero Effort, type I) ${ }^{6-11}$ where the modulation of the beam is performed before the sample. Therefore, in contrast to NSE, the MIEZE module we describe may even be used under depolarizing conditions in or

${ }^{\text {a)}}$ Electronic mail: robert.georgii@frm2.tum.de. around the sample. This routinely allows neutron scattering studies with the highest possible energy resolution in a wide range of materials.

Qualitatively, the MIEZE-I technique is based on a harmonic intensity modulation of the neutron beam, where the contrast $C$ is given by the ratio of the amplitude $A$ to the average signal $B$ as shown in Fig. 2(a). Using a phase-locked pair of two resonance spin flippers, ${ }^{9,13}$ which operate at slightly different frequencies, $\nu_{1}$ and $\nu_{2}$, induces a slow rotation of the polarization direction of the neutrons, which is subsequently converted into an intensity beating by means of a polarizing analyzer.

While former experiments with the MIEZE-I technique have been successful, we managed to implement MIEZE-I as a routine technique through a redesign of the neutron resonance spin flippers. Instead of wire-wound $\mathrm{B}_{0}$ coils we use electroerosion machined coil windings where a better definition of the magnetic field boundaries is obtained. They consist of a specially selected $\mathrm{Al}$ alloy with much less small angle scattering and a higher transmission. ${ }^{13}$ Together with a more reproducible mounting of the $\mathrm{rf}$ coils this results in

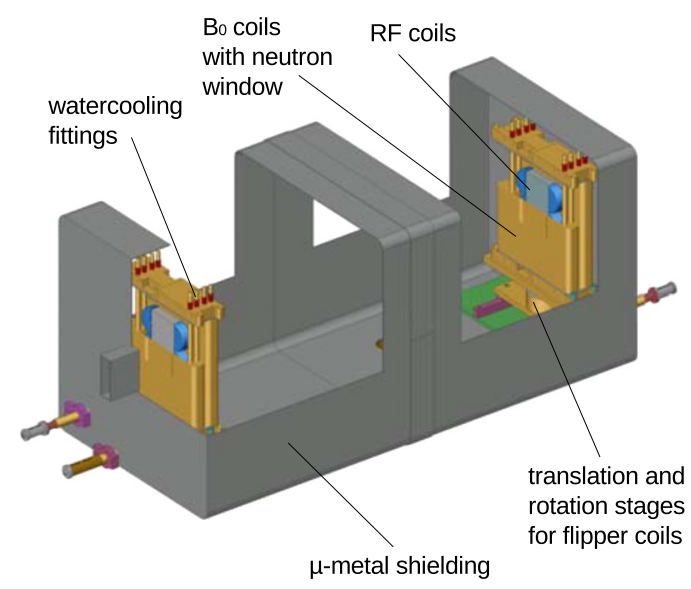

FIG. 1. (Color online) The "MIEZE box" as used for the MIEZE setup at MIRA and CG-1D at HFIR. 


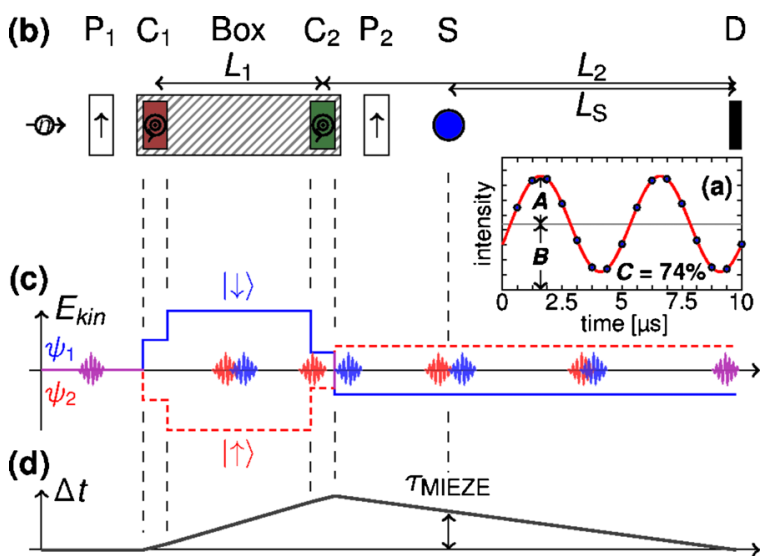

FIG. 2. (Color online) (a) A typical MIEZE signal at the detector position (see text for details). (b) Schematic of a complete MIEZE setup, showing the polarizer $\left(\mathrm{P}_{1}\right)$, the zero field of the MIEZE box (hatched) with two $\pi$-flipper coils $\left(\mathrm{C}_{1}, \mathrm{C}_{2}\right)$, the analyzer $\left(\mathrm{P}_{2}\right)$, the sample $(\mathrm{S})$ and the detector (D). (c) Kinetic energy splitting for the spin-down $\left(\psi_{1}\right)$ and spin-up $\left(\psi_{2}\right)$ states of the neutrons along the flight path due to the $\pi$-flipper coils. (d) Temporal delay $\Delta t$ of the spin states along the flight path. The splitting reaches its maximum after the second flipper coil and vanishes at the detector position.

stable $\pi$-flips over a wide range of rf frequencies with low small angle scattering background.

A description of the MIEZE-I principle alluding to similarities with time-of-flight methods is illustrated in Fig. 2; for a proper quantum mechanical description we refer to the literature (cf. Ref. 14). When a neutron arrives at the first spin flipper with its polarization perpendicular to the static field in the flipper, the correlation volumes (or wave-packages) corresponding to the spin-up and spin-down spin states are prepared [cf. Fig. 2(c)]. While the kinetic energy of the spindown state increases, the kinetic energy of the spin-up state decreases. Therefore, the correlation volumes for the spindown and spin-up states arrive at different times at the second resonance spin flipper placed at a distance $L_{1}$ behind the first spin flipper. This second spin flipper inverts the energy splitting of the spin states, reducing the kinetic energy of the spin-down state and increasing the kinetic energy of the spin-up state. Therefore the correlation volumes overlap again at a distance $L_{2}$ behind the second spin flipper, given by $L_{2}=L_{1}\left(\nu_{2} / \nu_{1}-1\right)^{-1}$ where $\nu_{2}>\nu_{1}$.

An analyzer at an arbitrary position between the second spin flipper and the detector (the latter is located where the correlation volumes meet) projects out the intensity of the interference pattern of the spin-up and spin-down states. As the correlation volumes of the spin-up and spin-down states have different energies, the interference pattern exhibits the intensity modulation of contrast $C$ referred to above.

To explain how this intensity modulation may be exploited in experimental studies using two single neutron resonance spin flippers, we show in Fig. 2(d) the delay between the two correlation volumes, $\Delta t$. The correlation volumes probe the sample at different times with the delay given by ${ }^{15} \tau_{\text {MIEZE }}=2 \cdot \hbar(2 \pi \Delta \nu) L_{S} /\left(m v^{3}\right)$, where $2 \Delta \nu=2\left(\nu_{2}\right.$ $\left.-\nu_{1}\right)$ is the frequency of the resulting MIEZE signal, $L_{S}$ is the distance between sample and detector, and $m$ and $v$ are the mass and average velocity of the neutrons. By overlapping these volumes at the detector, one obtains a signal contrast $C$ which is directly proportional to the intermediate scattering function $S\left(q, \tau_{\text {MIEZE }}\right)$, i.e., the information on the

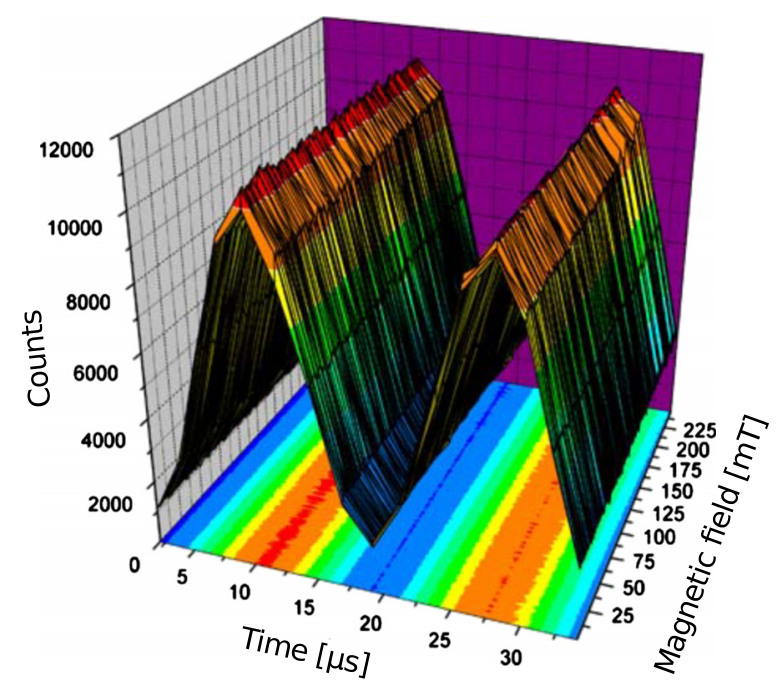

FIG. 3. (Color online) The MIEZE signal for the direct beam and a magnetic field produced with an electromagnet (Ref. 13). Note that the contrast does not depend on the field strength.

dynamics on this time scale. Further, for quasielastic scattering with an assumed Lorentzian line shape with half-width $\Gamma$, the normalized intermediate scattering function is given by $^{9} S(q, \tau) / S(q, 0)=\exp [-\Gamma(q) \tau]$, where $S(q, 0)$ corresponds to the intermediate scattering function of a purely elastically scattering sample.

The MIEZE-I technique is similar to conventional neutron resonance spin echo (NRSE) methods, where the two spin flippers before the sample correspond to the first arm of an NRSE instrument. Moreover, the MIEZE time $\tau_{\text {MIEZE }}$ is equivalent to the spin echo time in NSE and NRSE instruments. ${ }^{9}$ However, there is a distinct difference between MIEZE-I and NSE/NRSE. Placing the polarizing analyzer behind the second spin flipper and before the sample, the MIEZE-I technique becomes insensitive to effects of the sample or sample environment on the polarization of the neutron beam, i.e., for example, depolarization or applied magnetic fields. This is demonstrated in Fig. 3, where the contrast of the direct beam is plotted versus a magnetic field up to $0.2 \mathrm{~T}$ and no effect on the signal contrast can be observed.

The MIEZE experiments reported here were carried out at the diffractometer MIRA at FRM II using neutrons with a wavelength $\lambda=10.4 \AA \pm 5 \%$, i.e., a mean velocity $v$ $=380 \mathrm{~m} / \mathrm{s}$. The first and second spin flipper being $0.9 \mathrm{~m}$ apart were operating at frequencies in the range $46 \mathrm{kHz}$ $<\nu_{1}<200 \mathrm{kHz}$ and $69 \mathrm{kHz}<\nu_{2}<300 \mathrm{kHz}$, respectively, providing a beating frequency in the range $46 \mathrm{kHz}<2 \Delta \nu$ $<200 \mathrm{kHz}$. The distance between the sample and detector was $860 \mathrm{~mm}$. Taken together MIEZE times could be accessed in a range $280 \mathrm{ps}<\tau_{\text {MIEZE }}<1230$ ps. A $0.3 \mathrm{~mm}$ thick ${ }^{6} \mathrm{Li}$ doped glass scintillator with a photomultiplier was used as a fast detector.

We note that the temporal and thus spatial separation of the spin-up and spin-down states makes MIEZE-I sensitive to path length differences between the first rf-flipper and the detector, e.g., due to the large divergence of the beam, the finite size of the sample, or the finite thickness of the neutron detector. However, for the wavelength of $10.4 \AA$, frequencies in the range $46-200 \mathrm{kHz}$ for the MIEZE signal, typical sample sizes of $10 \mathrm{~mm}$, and scattering angles of the order of 

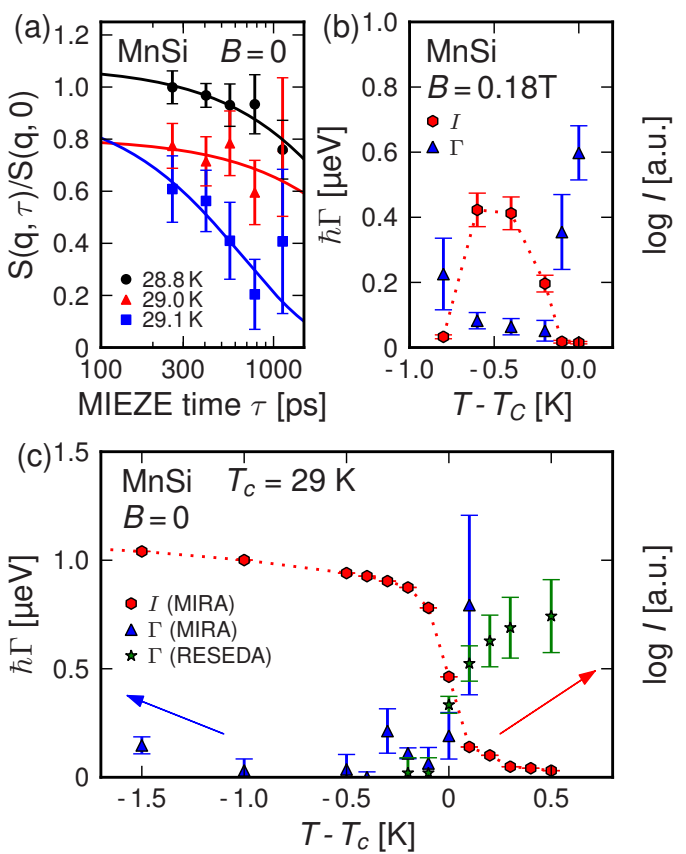

FIG. 4. (Color online) (a) Typical normalized intermediate scattering functions $S(q, \tau)$ in the helimagnetic state of $\mathrm{MnSi}$ at selected temperatures. (b) Line width $\Gamma$ in the A-phase of $\mathrm{MnSi}$ at $B=0.18 \mathrm{~T}$. The total scattering intensity is shown as solid hexagons, MIEZE data as solid triangles. The data are normalized to the line width of the helical phase at $T=3 \mathrm{~K}$. (c) Line width $\Gamma$ of the magnetic order in $\mathrm{MnSi}$ at $B=0$. The data are normalized to the line width at $T=3 \mathrm{~K}$. NRSE data from the instrument RESEDA are shown as stars.

a few degrees we measured with a standard sample used for resolution measurements a contrast reduction of less than $10 \%$ up to $q=0.05 \AA^{-1}$. This is in agreement with the results of calculations of the path length and precession phase differences.

To demonstrate the power of MIEZE-I in studies of field-induced forms of magnetic order normally not accessible to NSE/NRSE, we have investigated the cubic B20 compound $\mathrm{MnSi}^{16}{ }^{1} \mathrm{MnSi}$ orders helimagnetically below a transition temperature, in our case at $T_{c}=29.0 \mathrm{~K}$. The large pitch of the helix $\Lambda \approx 180 \AA$ implies that the associated magnetic Bragg peak at $q=0.035 \AA^{-1}$ can be accessed in a forward scattering configuration. Just below $T_{c}$ at $0.15 \mathrm{~T}$ $<B<0.2 \mathrm{~T}$, the A-phase is observed. Here a special form of magnetic order, a skyrmion lattice, is observed. ${ }^{17}$

Shown in Fig. 4(a) are typical data in the helimagnetic state $(B=0)$ of the normalized intermediate scattering function $S(q, \tau) / S(q, 0)$ for various temperatures. Data were normalized with respect to resolution measurements in the helical state at low temperatures, where the magnetic structure is supposed to be static. The solid lines are the result of fits to exponential functions. The presence of a second process on a shorter timescale as indicated by the fact that the fits are not converging to 1 for $\tau \rightarrow 0$ around $T_{c}$ is still under discussion and will be published elsewhere. The resulting line widths (solid triangles) as a function of temperature are shown in Fig. 4(c) (solid hexagons show the elastic magnetic intensity). While the magnetic order is resolution limited below $T_{c}$, there is broadening above $T_{c}$. For comparison, NRSE data from the same system are shown as stars.
The measured $\Gamma$ in the A-Phase of $\mathrm{MnSi}$ at $B=0.18 \mathrm{~T}$ [shown in Fig. 4(b)] is similar to the one in the helical phase. This demonstrates that even under applied magnetic fields the MIEZE-I technique may be readily used.

We have recently used our MIEZE box at the beam line CG-1D at HFIR at Oak Ridge National Laboratory. ${ }^{12}$ Here and on MIRA to set-up and adjust the MIEZE box requires less than a few hours making it indeed a turn-key measurement option.

In conclusion, we have developed a compact turn-key MIEZE module, that allows to improve the energy resolution of various neutron scattering instruments used for quasielastic scattering into the sub-micro-eV range for typical sample sizes of $1 \mathrm{~cm}$ in diameter and $q$ values up to $0.05 \AA^{-1}$. We have demonstrated the power of this technique by small angle scattering studies of the magnetic order of $\mathrm{MnSi}$ in applied magnetic fields. One particular strength of the MIEZE-I technique is its insensitivity to depolarizing conditions at the sample position, which is not easily achievable using standard spin echo techniques. This illustrates the wide range of scientific challenges that may now be addressed with sub-micro-eV resolution.

We gratefully acknowledge support by R. Schwikowski, A. Mantwill, M. Wipp, and the team of FRM II. We wish to thank R. Gähler, L. Robertson, I. Anderson, and W. Petry for helpful discussions and support. Financial support through the German Science Foundation (DFG) under TRR80, the Oak Ridge National Laboratory, and the European Commission under the 7th Framework Program, Contract no. CPCSA INFRA-2008-1.1.1 Number 226507-NMI3 is acknowledged.

${ }^{1}$ F. Mezei, Z. Phys. A 255, 146 (1972).

${ }^{2}$ R. Golub and R. Gähler, Phys. Lett. A 123, 43 (1987).

${ }^{3}$ C. Pappas, E. Lelievre-Berna, P. Bentley, E. Bourgeat-Lami, E. Moskvin, M. Thomas, S. Grigoriev, and V. Dyadkin, Nucl. Instrum. Methods Phys. Res. A 592, 420 (2008).

${ }^{4}$ C. Pappas, E. Lelievre-Berna, P. Falus, P. M. Bentley, E. Moskvin, S. Grigoriev, P. Fouquet, and B. Farago, Phys. Rev. Lett. 102, 197202 (2009).

${ }^{5}$ B. Farago and F. Mezei, Physica B 136, 100 (1986).

${ }^{6}$ R. Gähler, R. Golub, and T. Keller, Physica B 180-181, 899 (1992).

${ }^{7}$ M. Köppe, P. Hank, J. Wuttke, W. Petry, R. Gähler, and R. Kahn, J. Neutron Res. 4, 261 (1996).

${ }^{8}$ W. Besenböck, R. Gähler, P. Hank, R. Kahn, M. Köppe, C. H. D. Novion, W. Petry, and J. Wuttke, J. Neutron Res. 7, 65 (1998).

${ }^{9}$ T. Keller, R. Golub, and R. Gähler, Scattering and Inverse Scattering in Pure and Applied Science (Academic, London, 2002), pp. 1264-1286.

${ }^{10}$ M. Bleuel, M. Bröll, E. Lang, K. Littrell, R. Gähler, and J. Lal, Physica B 371, 297 (2006).

${ }^{11}$ Y. Kawabata, M. Hino, M. Kitaguchi, H. Hayashida, S. Tasaki, T. Ebisawa, D. Yamazaki, R. Maruyama, H. Seto, M. Nagao, and T. Kanaya, Physica B 385-386, 1122 (2006).

${ }^{12}$ G. Brandl, M. Bleuel, L. Robertson, L. Crow, J. Lal, and R. Georgii (unpublished).

${ }^{13}$ N. Arend, Ph.D. thesis, Technical University of Munich, 2007.

${ }^{14}$ N. Arend, R. Gähler, T. Keller, R. Georgii, T. Hils, and P. Böni, Phys. Lett. A 327, 21 (2004).

${ }^{15}$ The prefactor 2 is inherent to the NRSE/MIEZE technique when using the resonance flippers in the nonbootstrap mode.

${ }^{16}$ Y. Ishikawa, K. Tajima, D. Bloch, and M. Roth, Solid State Commun. 19, 525 (1976).

${ }^{17}$ S. Mühlbauer, B. Binz, F. Jonietz, C. Pfleiderer, A. Rosch, A. Neubauer, R. Georgii, and P. Böni, Science 323, 915 (2009). 